\title{
Top Five Artificial Intelligence Trends Affecting Leadership \& Management
}

\author{
Zoya Salmon-Powell \\ Global Projects Consultancy
}

\author{
Joya Scarlata \\ InterraIT
}

\author{
Edward F. Vengrouskie \\ Ft Hays State University
}

\begin{abstract}
Artificial Intelligence (AI), or sometimes referred to as machine learning, has become, over the last several years, a driving force in conducting business and has re-shaped the best practices across multiple industries, from retail to transportation. In addition, it has influenced significant innovations across the globe, including voice assistants, self-driving vehicles, and chatbots. This article explores how AI is impacting the business landscape, specifically in leadership and management. Trends discussed in this article are Robot Process Automation, Cyber Security, Data Accuracy, Customer Service, and Human Resources. Additional benefits of AI in the workplace include increased productivity, a greater focus on strategy, innovation, and improved organizational processes.
\end{abstract}

Keywords: artificial intelligence, leadership trends, management, workplace productivity

\section{INTRODUCTION}

The way we conduct business across industries is constantly evolving due to the economic, social, political, and technological changes. Leaders and managers alike are challenged more than ever to pivot quickly and adapt to the world's many changes. To stay ahead, they have to become agile, mobilize and motivate their teams around common goals that will add value to their customers and result in positive disruptions.

One such global influence that is shifting the business landscape is Artificial Intelligence (AI). AI refers to the simulation of human intelligence in machines programmed to think like humans and mimic their actions. It applies to any device that exhibits traits associated with the human mind, such as learning and problem-solving (Frankenfield, 2021, para.1). By 2030, AI will contribute up to $\$ 15.7$ trillion to the global economy, more than China and India's output combined (PwC, n.d. para. 4).

It is imperative for companies to get creative, flexible, and innovative, or risk being left behind. This article seeks to explore and share some of the top trends in AI affecting Leadership and Management across multiple fields and industries. Trends can lead to improving organizational efficiencies and help to transform business operations. 


\section{TREND \#1: ROBOT PROCESS AUTOMATION}

AI has played a pivotal role in reducing repetitive mundane work that managers and their teams perform. As a result, it has freed up time to focus on formulating and executing strategy, interpersonal tasks, and creative imagination.

Based on AI technology advancements, software-based robots are now handling basic tasks, such as filling out forms, generating diagrams, and producing documentation (Aggarwal, 2020). For example, the partnership between Tableau and Narrative Science, which resulted in Narratives for Tableau, creates explanations for Tableau graphics (Kolbjernsrud, Amico \& Thomas, 2016).

When companies can automate their business processes, it allows for less human error and wasted time. It fosters better collaboration as the team can readily identify their roles and actions. It also makes the task of reviewing and optimizing business processes less daunting and time-consuming.

\section{TREND \#2: AI IN CYBER SECURITY}

With many people practicing social distancing during this pandemic and using the online medium to conduct business, cybersecurity risks are at an all-time high. Many companies are investing large amounts of money in AI to prevent cyberattacks such as hacking and phishing. AI advanced prediction algorithms and smart technology play an essential role in protecting us from various nefarious attacks in our day-today lives (Aggarwal, 2020). Based on the pace at which e-commerce is rising, cyberattacks will continue to be a risk. Leaders must take the necessary steps to thoroughly assess the business and its technological infrastructure to identify the gaps and make the required improvements.

\section{TREND \#3: AI IN DATA ACCURACY}

Another common buzzword is "Data." All things data is on the lips of business professionals in many forums across the world. How companies maintain, store, and mine their information makes an enormous difference between companies that thrive and those that perish.

Companies must maintain quality and current data to access and leverage to make better business decisions readily. AI is playing a vital role in ensuring that companies are efficiently meeting that goal. AIdriven automated decision-making has improvised the accuracy of data over recent years. AI technology has been working on data simulations to ensure that it is correct and fit for interpretation purposes, informing companies' decisions (Aggarwal, 2020).

\section{TREND \#4: AI IN CUSTOMER SERVICE}

How we engage and interact with customers is changing due to the advancements in AI technologies. Chatbots are commonly being used on websites and elsewhere to guide the customer journey and address customer queries. Based on how intelligent the technology is, customers can interact with a chatbot and not realize that they are not speaking with humans.

With the advent of deep learning and semi-supervised models of machine learning, such as reinforcement learning, the algorithms will try and match up speech patterns and infer meaning from our natural human language (Aggarwal, 2020). Google Assistant is a prime example of how human interaction is not readily missed based on the deep programming done to mimic interaction with an actual human.

\section{TREND \#5: AI IN HUMAN RESOURCES}

The HR field is looking towards a future where leaders can optimize both automated and human work - this is where AI comes into play. As AI continues integrating into HR practices, organizations and their respective HR teams are empowered to make better and more informed decisions concerning prospective 
candidates and current employees (Nicastro, 2020). Also, AI plays a transformative role in HR practices, including training, onboarding, recruitment, retention, and performance analysis. An example of AI in HR is Koru, which tracks a potential applicant's credentials, qualities, and experiences. Using the available data, it helps to determine an appropriate fit between potential employers and job candidates for a specific position (VelocityGlobal, 2019).

\section{CONCLUSION}

From retail to transportation, industries are becoming immersed in AI in today's global economy. While some might view AI as a menacing threat, it is indeed possible for humans and machines to work side-byside, cooperatively, and in sync. There are benefits of making these technological investments in the short run for organizations as they help reduce operational expenses in the long run. By tapping into AI technologies, leaders and managers alike can unlock endless ways and methods to improve business outcomes, enhance operational efficiencies and other processes, and use data to augment decision-making and analysis. It will be exciting to see what AI will offer us over the next few years, and we are eager to highlight and share these new developments with you.

\section{REFERENCES}

Aggarwal, R. (2020, February 26). Top 10 AI Trends to Watch Out in 2020. Retrieved from https://datafloq.com/read/top-10-ai-trends-to-watch-out-2020/7813

Chamaki, F. (2018, May 27). Data Has a Better Idea. Retrieved from https://unsplash.com/photos/1K6IQsQbizI

Conick, H. (2017). The Past Present and Future of AI in Marketing. Retrieved from https://www.ama.org/marketing-news/the-past-present-and-future-of-ai-in-marketing

Frankenfield, J. (2021, March 8). Artificial Intelligence (AI). Retrieved from https://www.investopedia.com/terms/a/artificial-intelligence-ai.asp

Kolbjernsrud, V., Amico, R., \& Thomas, R.J. (2016, November 2). How Artificial Intelligence Will Redefine Management. Retrieved from https://www.pega.com/system/files/resources/201805/hbr-how-ai-will-redefine-management.pdf

Nicastro, D. (2020, May 18). 7 Ways Artificial Intelligence is Reinventing Human Resources. Retrieved from https://www.cmswire.com/digital-workplace/7-ways-artificial-intelligence-is-reinventinghuman-resources/

OECD. (2020). The Impact of Big Data and Artificial Intelligence (AI) in the Insurance Sector. Retrieved from www.oecd.org/finance/Impact-Big-Data-AI-in-the-Insurance-Sector.htm

pwc. (n.d.). Exploiting the AI Revolution. Retrieved from https://www.pwc.com/gx/en/issues/data-andanalytics/publications/artificial-intelligence-study.html

Smith, R.G. (2000). The Road Ahead for Knowledge Management: An AI Perspective. AI Magazine, 21(4), 17-40.

Velocity Global. (2019 March 21). 3 Examples of AI in HR in 2019. Retrieved from https:/velocityglobal.com/blog/3-examples-of-ai-for-hr-in-2019/ 